\title{
Implementation of reactive compensator for voltage balancing using AI based models and novel performance index
}

\author{
Dana Ragab ${ }^{1}$, Jasim Ghaeb ${ }^{2}$ \\ ${ }^{1}$ Mechatronics Engineering Department, Palestine Technical University-Kadoorie, Tulkarem, Palestine \\ ${ }^{2}$ Electrical Engineering Department, Philadelphia University, Amman, Jordan
}

\begin{tabular}{l} 
Article Info \\
\hline Article history: \\
Received Aug 16, 2021 \\
Revised Jan 24, 2022 \\
Accepted Jan 31, 2022 \\
\hline
\end{tabular}

Keywords:

Neural network

Power quality

Radial basis functions networks

Space vector

Thyristor controlled reactor

Voltage unbalance

\begin{abstract}
Voltage-unbalance is one of the power quality deficiencies that degrades electrical power systems performance. In this work, voltage unbalance problem is tackled through two stages; evaluation using a novel performance index and mitigation using a thyristor-controlled reactor (TCR) compensator with artificial intelligent (AI) based models. Unlike standard performance indices that rely on voltages' root mean square (RMS) values, the proposed index depends on the space vector (SV) signal amplitude for voltage unbalance evaluation. This signal depends on the instantaneous values of the three-phase voltages and has twice the system frequency. Therefore, the proposed index entitled as space vector unbalance factor (SVUF) reflects the amount of voltage unbalance and reduces the time necessary for evaluation by half. Subsequently, advanced models based on several algorithms are proposed to generate the required firing angles for TCR compensator to restore voltage balance, including radial basis functions networks (RBFNs), hybrid-RBFNs (H-RBFNs), polynomials (PNs), and simplified neural networks (NNs). Models' structure, prediction capability, and response time are analyzed. Results show that the time required for voltage unbalance mitigation is reduced. Moreover, the models used to generate the firing angles are simplified significantly while maintaining high accuracy.
\end{abstract}

This is an open access article under the CC BY-SA license.

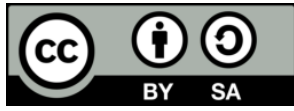

\section{Corresponding Author:}

Dana Ragab

Mechatronics Engineering Department, Palestine Technical University-Kadoorie

Jaffa St, Tulkarem, Palestine

Email: dmrafat@gmail.com

\section{INTRODUCTION}

Generation, transmission, and utilization of electrical power are critical issues prone to several power quality defects. Voltage unbalance is one of these problems which deteriorates various sectors performance [1]. The three-phase power system is considered balanced if the three-phase voltages and currents are symmetrical with a phase shift of $120^{\circ}$ [2].

The prime factor that leads to voltage unbalance is distributing the single-phase loads irregularly over the three-phase system. Moreover, unbalance could result from the asymmetry in transmission lines' impedances and transformer windings [3]. Voltage unbalance has severe effects on different devices, such as alternating current (AC) to direct current (DC) converters, adjustable speed drives, and induction motors [4]-[7]. Based on the degree of voltage unbalance, a derating factor must be used in determining the motor size; for instance, $12 \%$ larger motor is demanded in case of a $3 \%$ voltage unbalance [8]. Therefore, quantifying voltage unbalance is essential for evaluating system performance and employing a suitable control. To fulfill this requirement, the engineering community defined several indices to evaluate voltage unbalance. 
The true representation of voltage unbalance, was stated by international electrotechnical commission (IEC) as the ratio between the negative and positive sequence voltages. This index is known as voltage unbalance factor (VUF) [9]. Institute of Electrical and Electronics Engineers (IEEE) approved three definitions for voltage unbalance. According to IEEE Std.936-1987, voltage unbalance is expressed as "the difference between the highest and the lowest RMS phase voltage, referred to the average of the three voltages" [10]. Additionally, IEEE Std.112-1991, introduced phase voltage unbalance rate (PVUR), which is defined as the maximum deviation from the average of phase voltages divided by this average [11]. Finally, IEEE Std. 1159 confirmed the definition presented by IEC in addition to IEEE Std.112 [12]. Furthermore, national electrical manufacturers association (NEMA) authenticated another standard index known as line voltage unbalance ratio (LVUR). It is defined as the ratio between the maximum deviation from the average of line voltages and this average [13]. All these indices rely on the calculation of the RMS voltages therefore $20 \mathrm{~ms}$ is required at least to calculate them.

Moreover, several publications introduced other factors to evaluate or assess the effect of voltage unbalance on the system. Three indices, namely, maximum current deviation (MCD), combined current deviation (CMCD), and effective current deviation (ECD), were proposed in [14]. Henriques and Cormanein [15], voltage unbalance was evaluated in the time domain, and voltage unbalance level (VUL) performance index was proposed. This index requires many steps for calculation based on the second-order voltage tensor theory.

The subsequent step when unbalance exceeds the allowable limit which is $3 \%$ according to American National Standards Institute (ANSI) standard is voltage unbalance mitigation [16]. Many techniques were proposed in the literature for this purpose, in [17] dynamic reconfiguration was used to balance the distribution system by changing the distribution network structure. Other techniques that employ dynamic voltage restorer or a unified power conditioner to restore voltage were proposed in [18] and [19]. Another efficient technique is the use of flexible AC transmission system (FACTS) devices. These devices include static VAR compensators (SVC) and static synchronous compensators (STATCOM), which are widely used in voltage balancing [20]-[22]. Esfahani and Vahidi in [23], a combination of thyristorcontrolled reactor (TCR) and STATCOM was implemented to restore voltage balancing and reduce current harmonics.

Several control algorithms were proposed for SVC control [24]. The use of a PI controller with SVC for voltage regulation purposes was discussed in [25]. Hybrid algorithms that use neural networks (NNs) during online mode for voltage balancing and other algorithms to generate the firing angles of the TCR compensator during offline mode were considered [26]-[28]. A fuzzy ranking system was used in [26] to provide the optimum set of firing angles based on harmonic minimization. Rubaiey and Rubayi [27], a gravitational search algorithm (GSA) replaced the fuzzy logic with the same objectives of reducing harmonics and restoring voltage balancing. Particle swarm optimization (PSO) algorithm with the objective function of reducing VUF was proposed in [28]. Ragab et al. [29], proposed the NN was implemented during the online mode to retrieve voltage balance, while data required for NN training were obtained using an experimentally.

Radial basis functions networks (RBFNs) were used in [30] and [31] for SVC control, to improve the stability of the electrical power system. Guo et al. [32], used a model based on RBFNNs and principal component analysis (PCA) method was proposed to monitor the power system. The RRBFNN was suggested to deal with nonlinear data then the PCA was employed to perform islanding detection. Both NNs and RBFNs are good candidates to model complex engineering systems. The contribution to knowledge in this work can be summarized is being as:

- Proposing a novel index for voltage-unbalance evaluation. This index depends on the SV magnitude to calculate voltage unbalance percentage, reducing the time required for unbalance evaluation by half.

- Proposing several models to generate the required firing angles of TCR to restore voltage balance. Models based on RBFNs, hybrid-RBFNs (H-RBFNs), polynomials (PNs), and NN are proposed with the unbalanced three load voltages only as input data and the three firing angles of TCR as output data. The suggested NN has a very simple structure consisting of one hidden layer with ten neurons. Also, the proposed RBFNs and NN shows a high performance in prediction capabilities.

- Enhancing the response time required for voltage unbalance mitigation through improvements in both the evaluation and control process.

The paper is organized is being as: the unbalanced three phase power system is introduced in section 2. Then space vector unbalance factor (SVUF) is proposed for voltage unbalance evaluation in section 3. After that section 4 discusses the suggested models for voltage unbalance control. In section 5 the contribution to knowledge in this work is elaborated. Finally, the conclusion is given in section 6. 


\section{UNBALANCED THREE PHASE POWER SYSTEM}

Three-phase power systems operate efficiently under balanced conditions. However, voltageunbalance arises in several situations and degrades system performance. In this work, Aqaba Qatrana South Amman (AQSA) electrical power system is modeled and simulated in MATLAB/Simulink, as shown in Figure 1. The system's total length is $328 \mathrm{~km}$, starting at Aqaba station, which operates at $14 \mathrm{kV}$. This voltage is stepped up to $400 \mathrm{kV}$ and connected to the power system line. Two substations split the line; Qatrana substation (bus 2) at $245 \mathrm{~km}$ and Amman South substation (bus 3) at $328 \mathrm{~km}$, respectively. In order to represent these transmission lines, three pi-sections are used; each consists of two shunt capacitors and series inductor and resistor [29].

At South Amman substation, the voltage is stepped down to 132, 33, 11, and $0.38 \mathrm{kV}$ at which the three-phase loads are connected. As the power consumption by the loads is asymmetrical over the three phases, voltage unbalance arises. In this case, the currents drawn by the loads are not identical, producing asymmetrical voltage drop across the transmission line and consequentially unbalanced load voltages. In order to restore voltage balance, a TCR compensator is installed at bus 3. This type of reactive power compensator provides a good capability for individual phase control and fast response. By varying the firing angles between $90^{\circ}$ and $180^{\circ}$, the absorbed reactive power varies accordingly. In the case of voltage unbalance, the provided lagging VARs differ according to each phase requirements, and thus the control action can be carried out by firing each pair of thyristors with proper firing angles [20]. The required firing angles are determined using several algorithms, as discussed in section 4.

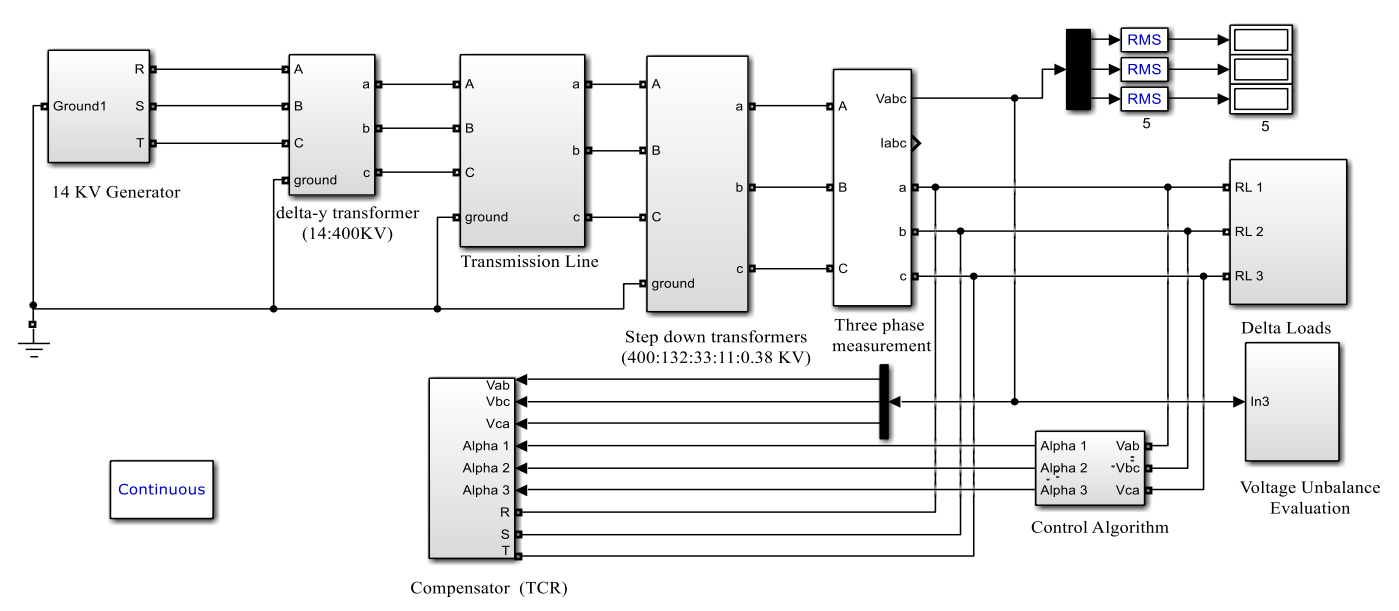

Figure 1. AQSA power system Simulink model

\section{VOLTAGE UNBALANCE EVALUATION USING SVUF}

In this section standard indices used for voltage unbalance evaluation are introduced, and the new SVUF index is proposed and compared to these indices.

\subsection{Voltage unbalance factor}

The symmetrical components theory can be used to analyze and study the power system under unbalanced conditions [33]. In the context of this work, it is used as a part of VUF calculation. According to this theory, the three-phase unbalanced components can be expressed as a set of balanced (i.e., symmetrical) ones. These symmetrical components are known as positive, negative, and zero sequence components. Both the positive and negative sequence voltages have equal magnitudes and a phase shift of $120^{\circ}$. But the negative sequence voltages rotate in the opposite direction of positive sequence voltages. The zero-sequence voltages have equal magnitudes, zero phase shift, and fixed (i.e., do not rotate). The symmetrical components can be represented in terms of the three-phase voltages is being as [33]:

$$
\begin{aligned}
& V_{0}=1 / 3\left(V_{a}+V_{b}+V_{c}\right) \\
& V_{1}=1 / 3\left(V_{a}+a V_{b}+a^{2} V_{c}\right) \\
& V_{2}=1 / 3\left(V_{a}+a^{2} V_{b}+a V_{c}\right)
\end{aligned}
$$

Where: 
$V_{a}, V_{b}$ and $V_{c}$ : Three-phase unbalanced voltages.

$V_{0}, V_{1}$ and $V_{2}$ :Zero, positive, and negative sequence components of the three-phase voltages, respectively.

The VUF, which represents the true definition, can be calculated according to the following equation:

$$
\mathrm{VUF}=\frac{\mathrm{V}_{2}}{\mathrm{~V}_{1}} \times 100 \%
$$

\subsection{Phase voltage unbalance rate}

In terms of IEEE standards, the PVURs are given by (5) and (6) with subscripts referring to the standard's name [11], [12]. The PVUR is defined by IEEE- Std.112 as the maximum deviation from the average of three-phase voltages to the average of three-phase voltages. The IEEE- Std.936 defined PVUR as the difference between the highest and lowest voltage to the average of three-phase voltages. Its calculation depends on the RMS of the voltages, which requires $20 \mathrm{~ms}$ of time.

$$
\begin{aligned}
& P_{V U R_{112}}=\frac{\text { Maximum deviation from average }}{\text { Average of three phase voltages }} \times 100 \% \\
& P V U R_{936}=\frac{\Delta \text { highest and lowest rms voltage }}{\text { Average of three voltages }} \times 100 \%
\end{aligned}
$$

\subsection{Line voltage unbalance ratio}

In (7) represents the NEMA definition for LVUR [13]. Its calculation depends on the RMS of the voltages, which requires $20 \mathrm{~ms}$ of time.

$$
\text { LVUR }=\frac{\text { Maximum deviation from average }}{\text { Average of three line voltages }} \times 100 \%
$$

\subsection{Space vector unbalance factor}

In this work, an advanced index to evaluate voltage unbalance based on SV signal is proposed. Space vector unbalance factor (SVUF) is the suggested name for the proposed index. In general, the SV is a complex variable used to represent the total effect of system voltages, as shown by (8) [29]. When the three-phase power system is balanced, the SV magnitude is constant and can be considered as a DC signal. Whereas, when the three-phase voltages are unbalanced, the instantaneous values of the SV magnitude vary continuously producing a sinusoidal signal. The frequency of this signal is twice the system voltages frequency [29].

$$
\bar{v}_{s}=v_{a}+j \frac{1}{\sqrt{3}}\left(v_{b}-v_{c}\right)
$$

The magnitude of SV $\left|\bar{v}_{S}\right|$ can be calculated by (9) [34].

$$
\left|\bar{v}_{s}\right|=\sqrt{v_{a}^{2}+\frac{1}{3}\left(v_{b}-v_{c}\right)^{2}}
$$

Where:

$v_{a}, v_{b}$, and $v_{c}$ : The three-phase load voltages.

The proposed SVUF is defined as the ratio between the amplitudes of the SV AC signal and the SV DC signal. Or "The ratio between the SV amplitudes under unbalanced and balanced conditions". Accordingly, SVUF can be calculated from (10).

$$
\% S V U F=\frac{V_{S(A C)}}{V_{S(D C)}} * 100 \%
$$

Where:

$V_{S(D C)}: \mathrm{SV}$ amplitude under balanced condition.

$V_{S(A C)}: \mathrm{SV}$ amplitude under unbalanced condition.

Figure 2 shows the three-phase voltages and SV amplitude for the considered AQSA system under balanced conditions. The SV amplitude equals $362 \mathrm{~V}$, which is taken as a reference DC value. Figure 3 shows the SV signal in case of voltage unbalance in which the VUF is $4.11 \%$. In this case, the amplitude of $\mathrm{SV}$ is $14.65 \mathrm{~V}$, and according to (10), the SVUF can be calculated is being as:

$\% S V U F=\frac{V_{S(A C)}}{V_{S(D C)}} * 100 \%=\frac{14.65}{362} * 100 \%=4.04 \%$. 
Figure 4 shows another case in which the VUF equals $1.567 \%$, and the SV amplitude is $5.634 \mathrm{~V}$, and accordingly, the SVUF is $1.556 \%$. The absolute error between the obtained SVUF and the real VUF is only $0.07 \%$ and $0.011 \%$, respectively. This confirms the accuracy of the proposed SVUF for the calculation of the unbalance factor in unbalanced three phase power system.

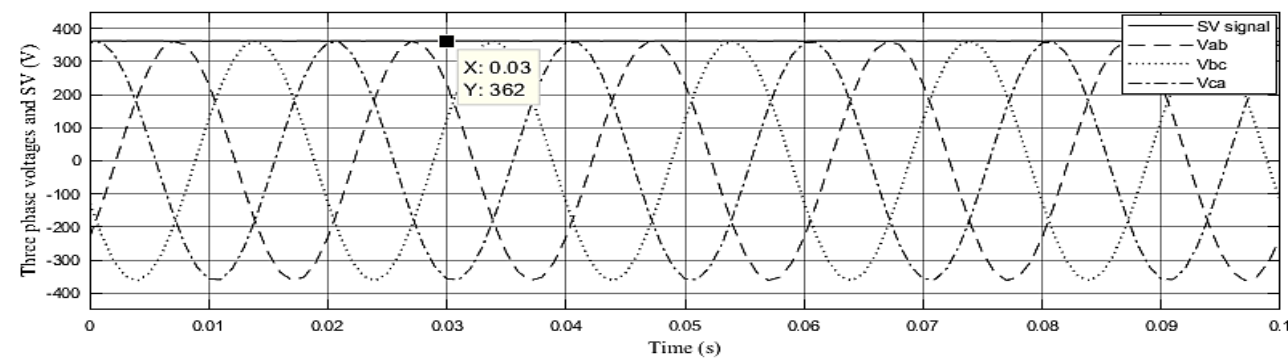

Figure 2. SV signal and balanced three-phase voltages

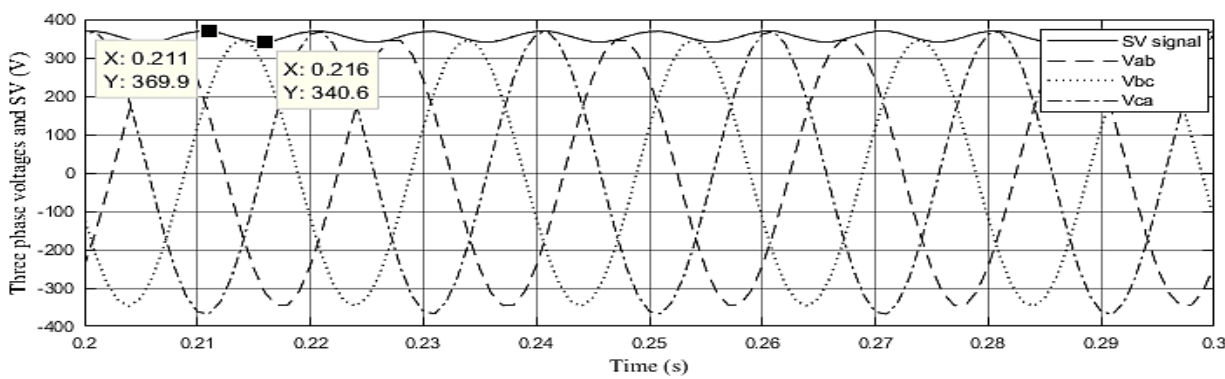

Figure 3. SV signal and unbalanced three-phase voltages with $\%$ SVUF of $4.04 \%$ and $\%$ VUF of $4.11 \%$

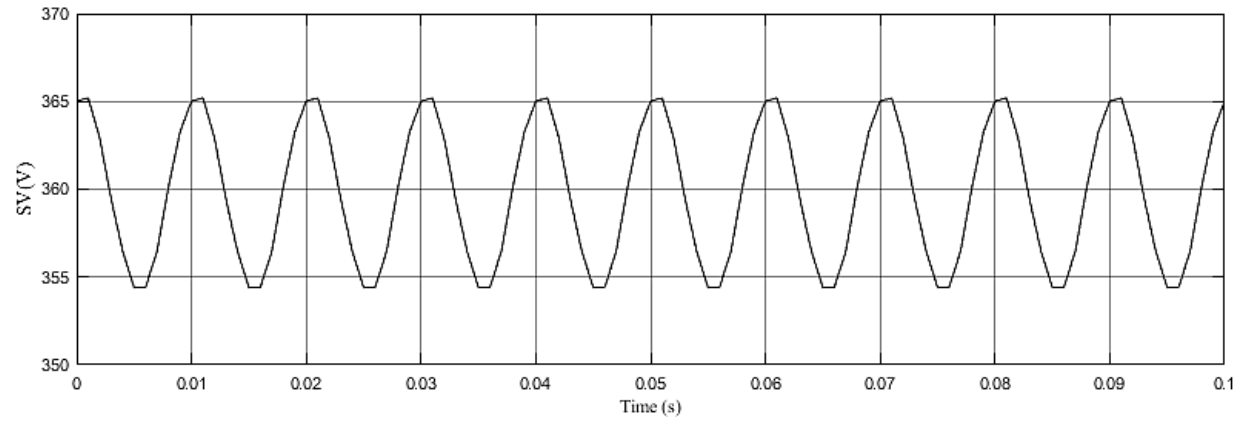

Figure 4. SV signal with \%SVUF of $1.567 \%$ and $\%$ VUF of $1.566 \%$

Table 1 shows the SVUF and the other standard indices (LVUF, PVUF ${ }_{112}$, and PVUF $_{936}$ ) in comparison with the true value which is represented by VUF. It can be seen that the values obtained using the proposed SVUF provide adequate representation for voltage unbalance percentage in the system. Furthermore, the average of absolute errors for each performance index is calculated, assuming that the $\%$ VUF represents the true value. It is noticeable that the proposed performance index SVUF provides the least average absolute error, which equals to $0.48 \%$.

The proposed index provides good accuracy while surpasses other indices in several aspects. It eliminates the need to express the system voltages in terms of the symmetrical components required for the VUF calculation. Moreover, the SV signal has twice the frequency of the system voltages signal, hence this signal's amplitude can be provided within half of the system cycle (i.e., $10 \mathrm{~ms}$ for $50 \mathrm{~Hz}$ signal frequency). While the RMS values of system voltages require $20 \mathrm{~ms}$ at least when unbalance conditions are presented in system. Consequently, the proposed index outdoes the traditional performance indices by reducing the time necessary to detect and evaluate voltage unbalance to half. 
Table 1. Proposed SVUF and standard indices in comparison with VUF

\begin{tabular}{cccccc}
\hline SV amplitude & $\% V U F$ & $\% S V U F$ & $\% L V U F$ & $\% P V U R_{112}$ & $\% P V U R_{936}$ \\
\hline 11.9 & 3.4 & 3.29 & 3.31 & 3.24 & 5.59 \\
8.405 & 2.38 & 2.32 & 2.36 & 2.35 & 3.88 \\
21.76 & 6.35 & 6.01 & 5.59 & 5.47 & 10.9 \\
20.05 & 5.83 & 5.54 & 5.19 & 5.85 & 9.93 \\
23.71 & 6.93 & 6.55 & 6.18 & 6.29 & 11.9 \\
39.17 & 12 & 10.8 & 10.5 & 10.5 & 20.6 \\
34.82 & 10.5 & 9.62 & 9.48 & 9.13 & 18.1 \\
32.19 & 9.68 & 8.89 & 8.73 & 8.44 & 16.6 \\
30.25 & 9.01 & 8.36 & 7.8 & 8.05 & 15.6 \\
28.3 & 8.37 & 7.82 & 7.45 & 7.68 & 14.4 \\
14.62 & 4.11 & 4.04 & 4.06 & 4.16 & 6.48 \\
Average absolute error & $0 \%$ & $0.48 \%$ & $0.72 \%$ & $0.69 \%$ & $5.04 \%$ \\
\hline
\end{tabular}

\section{VOLTAGE UNBALANCE MITIGATION}

In this work, a TCR reactive power compensator at the load side is applied to restore the voltage balance for the $328 \mathrm{~km}$ AQSA power system. By determining the firing angles of the TCR, the amount of reactive power can be controlled and balanced conditions can be achieved. In this section four intelligent models: RBFNs, H-RBFNs, PNs, and NNs are proposed to generate the required TCR firing angles. Then the best models are validated through the employment on the AQSA power system considering the VUF as the criterion for assessment. Finally, the two steps of voltage unbalance evaluation and mitigation using SVUF and intelligent models are performed, respectively.

All the proposed intelligent models utilize the three phase voltages as inputs to predict the required three firing angles for voltage balancing. A MATLAB/Simulink model of AQSA is used to generate the data set that are used to train the proposed intelligent models empirically. This procedure was followed to reduce the number of parameters used as the input data. Because equations required to calculate the firing angles depend on at least six parameters, including three load voltages, currents, real powers, and reactive powers, therefore using these equations to generate the data during offline mode then excluding part of them as in [27] and [28] affects the quality of data. Consequentially, NN's with complex structures were necessary to perform the regression with results showing moderate performance during the testing phase. 230 samples of data set are generated to train intelligent models, $80 \%$ of these samples are used for regression, and $20 \%$ for validation (i.e., testing). Different models are compared according to several factors is being as:

- The number of parameters constitutes the model.

- The coefficient of determination $\left(\mathrm{R}^{2}\right)$, which reflects the model's ability to produce the expected output, and the optimal value for this factor is 1 .

- The predicted error sum of squares $\mathrm{R}^{2}$ (PRESS $\mathrm{R}^{2}$ ), which reflects the model capability in prediction, and the optimal value for this factor is 1 .

- The root mean square error (RMSE), which indicates the model's ability to chase the data points, and the optimal value for this factor is zero.

- Predicted error sum of squares RMSS (PRESS RMSE), which helps in avoiding overfitting during model generation. As this factor decrease the model will have a better predectivity. Reducing the RMSE value does not guarantee that model will produce good results when new data are presented. Therefore, it is useful to consider minimizing both the RMSE and PRESS RMSE.

\subsection{Radial basis function networks}

Several types of RBFNs are considered to predict the firing angles. In general, the radial basis function can be represented by (11) [35].

$$
z(x)=\phi(\|x-\mu\|)
$$

Where $x$ represents the input vector and $\mu$ is the center of the radial bases function. In terms of $\phi$, it is a univariate function that characterizes RBFNs' various functions, which are known as kernel functions.

Each model obtained using RBFNs consists of linearly combined $N$ of RBFs that have distinct centers. The output of the model built is given by (12).

$$
\hat{y}(x)=\sum_{j=1}^{N} \beta_{j} z_{j}(x)
$$

Where $\hat{y}(x)$ is the approximated output of a target set and $\beta_{j}$ is the weight of the $\mathrm{j}^{\text {th }} \mathrm{RBF}$. 
In this work the kernel functions considered are: multiquadrics (MQ), thin-plate spline (TPS), and logistic basis function (LBF). These kernel functions are given by (13)-(15) respectively. All these functions have a width parameter $\sigma$ related to the function spread around its center.

$$
\begin{aligned}
& \phi(r)=(r / \sigma)^{2} \log (r / \sigma) \\
& \phi(r)=\frac{1}{1+\exp \left(\frac{r}{\sigma}\right)} \\
& \phi(r)=\sqrt{r^{2}+\sigma^{2}}
\end{aligned}
$$

Table 2 shows that all Kernel functions resulted in an outstanding performance in terms of $\mathrm{R}^{2}$, which has a value of 1 . Which proves the models ability to produce the expected output. Also, it can be seen that PRESS $\mathrm{R}^{2}$ is very high, almost 1 for all models, which manifests high performance in terms of predictability. Moreover, the least RMSEs are provided by MQ, LBF, and LBF. Therefore, a combination of these functions is applied in the Simulink model to perform voltage unbalance mitigation.

Table 2. RBFNs models results

\begin{tabular}{ccccccc}
\hline TCR firing angles & Kernel Function & Parameters & R $^{2}$ & PRESS R $^{2}$ & RMSE & PRESS RMSE \\
\hline$\alpha 1$ & MQ & 62 & 1 & 0.999 & 1.19 & 3.25 \\
& TPS & 63 & 1 & 0.999 & 1.38 & 3.75 \\
\multirow{4}{*}{$\alpha 2$} & LBF & 60 & 1 & 0.998 & 1.26 & 4.29 \\
& MQ & 63 & 1 & 0.999 & 1.91 & 4.36 \\
& TPS & 63 & 1 & 0.996 & 1.92 & 7.39 \\
\multirow{2}{*}{$\alpha 3$} & LBF & 63 & 1 & 0.999 & 1.85 & 3.97 \\
& MQ & 63 & 1 & 0.999 & 1.78 & 3.5 \\
& TPS & 63 & 1 & 0.999 & 1.57 & 3.49 \\
& LBF & 63 & 1 & 0.999 & 1.53 & 3.7
\end{tabular}

\subsection{Hybrid radial basis function networks}

These functions are a combination of RBFNs and linear models. The H-RBFNs considered are hybrid-LBF (HLBF), hybrid-MQ (HMQ), and hybrid-liner RBF (HLRBF) with PNs. Table 3 shows that a high value of $\mathrm{R}^{2}$ for the three firing angles of more than or equal to 0.99 is reached for all models. It shows the models ability to produce the expected output. Furthermore, PRESS $\mathrm{R}^{2}$ is acceptable and shows good prediction capabilities. In terms of least RMSE, it is accomplished by the LBFP model; hence it is used in section 4.5 for voltage unbalance mitigation.

Table 3. H-RBFNs models results

\begin{tabular}{ccccccc}
\hline TCR firing angles & H-RBF & Parameters & $\mathrm{R}^{2}$ & PRESS R $^{2}$ & RMSE & PRESS RMSE \\
\hline$\alpha 1$ & HLBF & 67 & 0.997 & 0.929 & 1.00 & 4.6 \\
& HMQ & 67 & 0.997 & 0.941 & 1.00 & 4.06 \\
\multirow{4}{*}{$\alpha 2$} & HLRBF & 67 & 0.997 & 0.945 & 1.10 & 4.18 \\
& HLBF & 67 & 0.99 & 0.971 & 1.59 & 3.54 \\
& HMQ & 67 & 0.99 & 0.977 & 1.65 & 4.98 \\
& HLRBF & 67 & 0.99 & 0.972 & 1.68 & 3.14 \\
& HLBF & 67 & 0.994 & 0.971 & 1.37 & 3.18 \\
& HMQ & 67 & 0.993 & 0.97 & 1.44 & 3.76 \\
& HLRBF & 67 & 0.994 & 0.96 & 1.49 & 3.28 \\
\hline
\end{tabular}

\subsection{Polynomials}

Linear regression provides simpler models compared to other regression types. Nevertheless, when dealing with PNs models, attention must be given to the number of terms. This number must be selected so that a compromisation between reducing the error and avoiding overfitting is achieved [35]. Regarding that, as the number of terms increases, the error decreases. To provide a better generalization (i.e., model predictability), stepwise regression is used to select model terms so that the PRESS RMSE is minimized.

Several polynomial models are examined to predict the firing angles. A third, fourth, and fifth-order polynomials are investigated, as shown in Table 4. It can be noticed that as the order of the polynomial increases and consequentially, the number of terms increases, the RMSE decreased slightly. However, the PRESS RMSE increased numerously and PRESS $\mathrm{R}^{2}$ decreased, reflecting a reduction in the prediction capability of the higher-order models and overfitting. As a result, the third-order polynomial will be considered for voltage unbalance control. 
Table 4. Polynomials models results

\begin{tabular}{ccccccc}
\hline TCR firing angles & Polynomial Order & Parameters & $\mathrm{R}^{2}$ & PRESS R $^{2}$ & RMSE & PRESS RMSE \\
\hline$\alpha 1$ & $3^{\text {rd }}$ & 15 & 0.964 & 0.931 & 4.32 & 4.51 \\
& $4^{\text {th }}$ & 35 & 0.966 & 0.215 & 3.5 & 15.27 \\
\multirow{2}{*}{$\alpha 2$} & $5^{\text {th }}$ & 38 & 0.968 & -10.695 & 3.46 & 58.94 \\
& $3^{\text {rd }}$ & 14 & 0.939 & 0.926 & 5.3 & 5.61 \\
$\alpha 3$ & $4^{\text {th }}$ & 35 & 0.972 & 0.623 & 3.86 & 10.81 \\
& $5^{\text {th }}$ & 38 & 0.973 & -13.155 & 3.77 & 77.81 \\
& $3^{\text {rd }}$ & 14 & 0.96 & 0.951 & 3.9 & 4.16 \\
& $4^{\text {th }}$ & 23 & 0.96 & -0.094 & 3.82 & 19.63 \\
\hline
\end{tabular}

\subsection{Neural networks}

Many publications in the literature discussed the use of $\mathrm{NN}$ for firing angles generation. Typically, NN's use is widespread during online operation due to its fast response compared to other algorithms such as gravitational search, particle swarm, and genetic algorithm that are usually used to generate the firing angles during offline mode [28]. Two structures of NN are suggested. First, three NNs are used with the three load voltages as input and one of the firing angles as each network's output. Each NN consists of one hidden layer with ten neurons and an output layer with one neuron; the tansigmoid transfer function is considered in both layers. Table 5 shows the data related to NNs training. It can be seen that in all cases, $\mathrm{R}^{2}$ is very high almost one, which means that the NNs have an excellent performance during the training phase.

In terms of the second structure, one NN with the three load voltages as input and the three firing angles as output is suggested. The NN consists of one hidden layer with ten neurons, which reduces calculations significantly. Repeatedly, the tansigmoid transfer function is used for both the hidden and the output layers. Figure 5 shows the performance of the proposed NN during the training, validation, and testing phases. Outstanding performance is achieved where $\mathrm{R}^{2}$ is more than 0.97 during all phases while attaining considerable simplification in the structure compared to other NNs applied for the same problem.

Table 5. Results of NN models

\begin{tabular}{cccc}
\hline TCR firing angles & Parameters & $\mathrm{R}^{2}$ & RMSE \\
\hline$\alpha 1$ & 51 & 0.97 & 3.27 \\
$\alpha 2$ & 51 & 0.95 & 5.16 \\
$\alpha 3$ & 51 & 0.979 & 3.01 \\
\hline
\end{tabular}
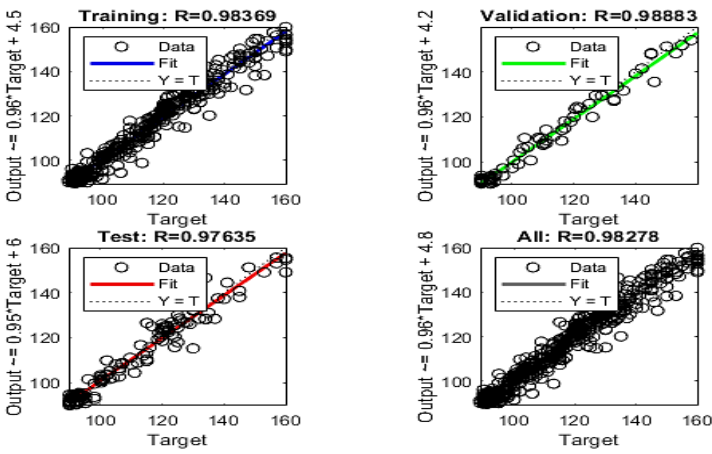

Figure 5. NN performance

\subsection{Models validation}

The best models obtained in the previous sections are validated through 33 test cases. These cases are conducted on the AQSA power system model using MATLAB/Simulink. A third-order polynomial, RBFN, H-RBFN, and NN are used to generate the required TCR firing angles for voltage balancing. As mentioned earlier, the acceptable value of the VUF is less than 3\%. According to this criterion, the proposed models are evaluated. Table 6 shows the unbalanced voltages and the VUF before and after correction using different models, for different load changes at AQSA. All models provide acceptable performance and achieve the goal of reducing VUF to less than 3\% except for polynomials, which screwed in some cases and generally maintained higher VUF. Also, it can be seen that the RBFN provides the best performance, with an average VUF of $0.76 \%$. This result can be elucidated by RBFN models' high performance in terms of PRESS $\mathrm{R}^{2}$ and $\mathrm{R}^{2}$, indicating these models' abilities to predict and fit data. 
Table 6. VUF using different models

\begin{tabular}{|c|c|c|c|c|c|c|c|}
\hline \multicolumn{5}{|c|}{ Before voltage unbalance mitigation } & \multicolumn{3}{|c|}{ After voltage unbalance mitigation } \\
\hline VUF & $\mathrm{V}_{\mathrm{ab}}$ & $\mathrm{V}_{\mathrm{bc}}$ & $\mathrm{V}_{\mathrm{ca}}$ & VUF (Polynomial) & VUF (H-RBFN) & VUF (RBFN) & VUF (NN) \\
\hline 3.68 & 225 & 234 & 219 & 0.36 & 0.33 & 0.49 & 1.01 \\
\hline 6.68 & 220 & 230 & 205 & 1.93 & 0.67 & 0.67 & 0.9 \\
\hline 4 & 225 & 233 & 217 & 0.15 & 0.26 & 0.33 & 1.07 \\
\hline 6.48 & 218 & 235 & 210 & 2.8 & 0.6 & 0.6 & 0.85 \\
\hline 4.5 & 219 & 230 & 213 & 0.88 & 0.4 & 0.36 & 0.4 \\
\hline 6.3 & 219 & 232 & 208 & 0.96 & 0.49 & 0.47 & 0.521 \\
\hline 6 & 216 & 232 & 210 & 1.23 & 0.96 & 0.7 & 0.37 \\
\hline 5.9 & 214 & 232 & 215 & 0.48 & 0.75 & 0.72 & 0.22 \\
\hline 5 & 222 & 236 & 217 & 0.4 & 0.66 & 0.64 & 0.78 \\
\hline 7.5 & 221 & 229 & 202 & 2.97 & 1.93 & 1.65 & 1.68 \\
\hline 3.37 & 227 & 238 & 224 & 0.55 & 0.45 & 0.34 & 1.5 \\
\hline 3.7 & 228 & 225 & 240 & 1.94 & 1.71 & 0.86 & 1.26 \\
\hline 3.9 & 229 & 222 & 238 & 1.65 & 0.774 & 1.06 & 1.07 \\
\hline 4.84 & 223 & 220 & 238 & 1.32 & 0.58 & 1.31 & 1.17 \\
\hline 6 & 223 & 218 & 240 & 2.8 & 0.36 & 0.92 & 0.85 \\
\hline 5.37 & 223 & 220 & 239 & 2 & 0.26 & 0.4 & 0.37 \\
\hline 7.11 & 222 & 216 & 242 & 3.89 & 1.06 & 1.71 & 1.65 \\
\hline 3.48 & 218 & 222 & 232 & 0.84 & 0.74 & 0.52 & 1.02 \\
\hline 3.47 & 216 & 214 & 226 & 1.16 & 0.25 & 0.17 & 0.7 \\
\hline 4.77 & 205 & 213 & 223 & 0.64 & 0.32 & 0.35 & 0.27 \\
\hline 6.3 & 200 & 210 & 223 & 1.4 & 1.5 & 1.13 & 1.1 \\
\hline 5.25 & 202 & 213 & 221 & 0.77 & 0.58 & 0.59 & 0.23 \\
\hline 4.29 & 202 & 209 & 218 & 0.95 & 0.55 & 0.47 & 0.8 \\
\hline 3.28 & 231 & 231 & 220 & 1.09 & 0.34 & 0.37 & 1.4 \\
\hline 5.05 & 226 & 232 & 214 & 0.21 & 0.14 & 0.23 & 0.29 \\
\hline 4.1 & 229 & 231 & 217 & 0.69 & 0.56 & 0.72 & 0.91 \\
\hline 6 & 227 & 230 & 208 & 1.66 & 1.39 & 1.06 & 0.08 \\
\hline 7.17 & 224 & 231 & 205 & 2.82 & 2.41 & 1.29 & 1.12 \\
\hline 6.9 & 224 & 227 & 203 & 1.9 & 1.845 & 0.91 & 0.67 \\
\hline 4.79 & 219 & 221 & 205 & 0.35 & 0.64 & 0.67 & 0.55 \\
\hline 5.94 & 221 & 221 & 204 & 1.11 & 0.85 & 0.71 & 0.49 \\
\hline 7.9 & 226 & 224 & 199 & 2.43 & 3.5 & 2.04 & 1.27 \\
\hline 3.99 & 229 & 227 & 215 & 0.6 & 0.3 & 0.52 & 0.19 \\
\hline Average VUF & 5.24 & & & 1.36 & 0.85 & 0.76 & 0.81 \\
\hline
\end{tabular}

Figure 6 shows the response of the three-load voltages at AQSA with 5.2\% voltage unbalance occurs at $0.08 \mathrm{sec}$. Then at $0.13 \mathrm{sec}, \mathrm{RBFN}$ is used to generate the required TCR firing angles $\left(99^{\circ}, 96^{\circ}\right.$, $130^{\circ}$ ) and the voltage unbalance is mitigated to $0.75 \%$. To further demonstrate the advantage of the proposed SVUF, two cases for voltage unbalance evaluation and mitigation are considered. In both cases, after voltage unbalance detection, $10 \mathrm{msec}$ are considered to calculate the three load voltages used as NN inputs. Next, the three firing angles are produced by the NN instantly. Figure 7 shows that the total time required to retrieve the balance condition is $30 \mathrm{msec}$ when VUF is used to evaluate the voltage unbalance. On the other hand, Figure 8 shows that this time is reduced to $20 \mathrm{msec}$ when SVUF is utilized for the evaluation. Therefore, the actual time required for voltage unbalance detection and evaluation when the VUF used is 20 msec, while in the case of SVUF, the time is reduced to $10 \mathrm{msec}$. It is worth to mention that in both cases voltage unbalance occurs at $0.1 \mathrm{~s}$. Figure 9 shows one of the line voltages Vca in both cases. The control action takes place at $0.12 \mathrm{~s}$ in the case of SVUF, while in the case of VUF, it takes place at $0.13 \mathrm{~s}$.

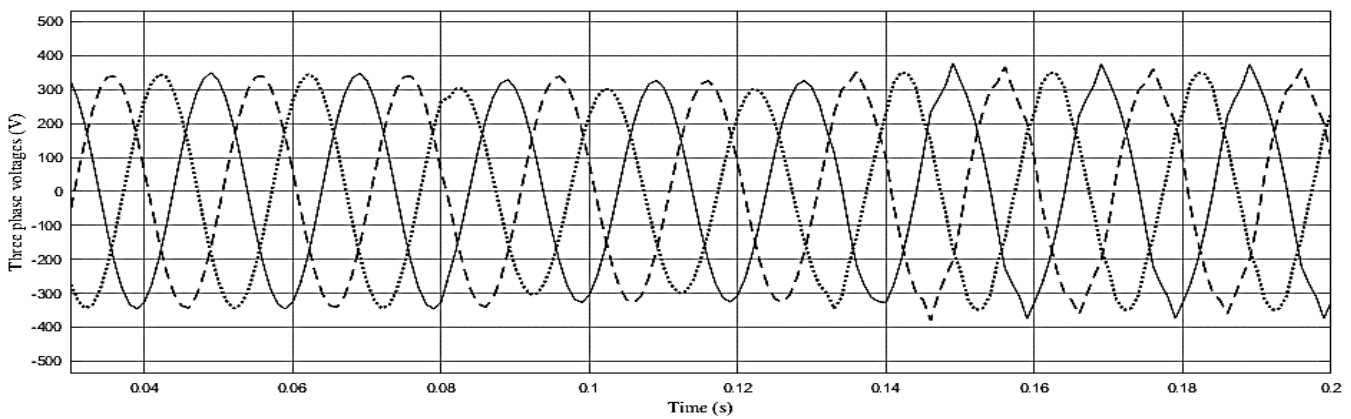

Figure 6 Three phase load voltages with RBFNs to retrieve voltage balancing 


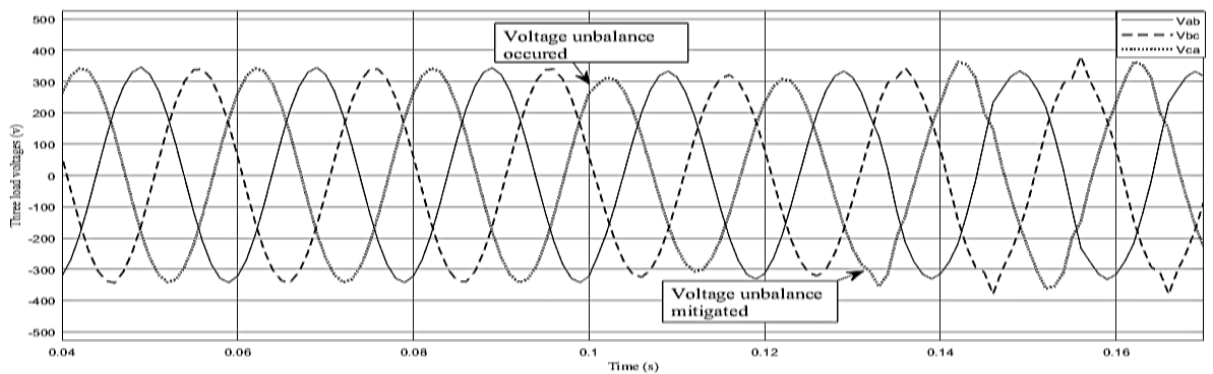

Figure 7. Voltage unbalance mitigation and evaluation using NNs and VUF

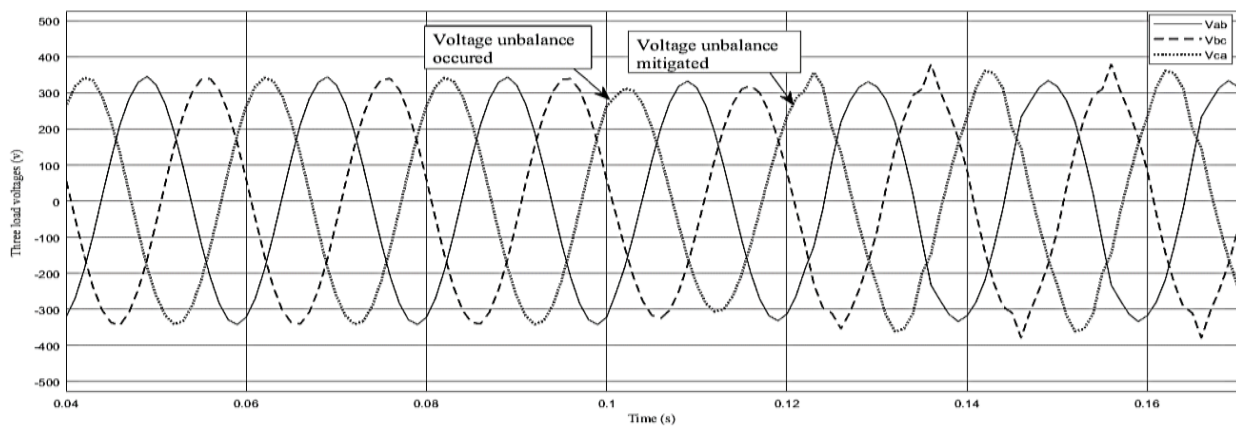

Figure 8. Voltage unbalance mitigation and evaluation using NNs and SVUF

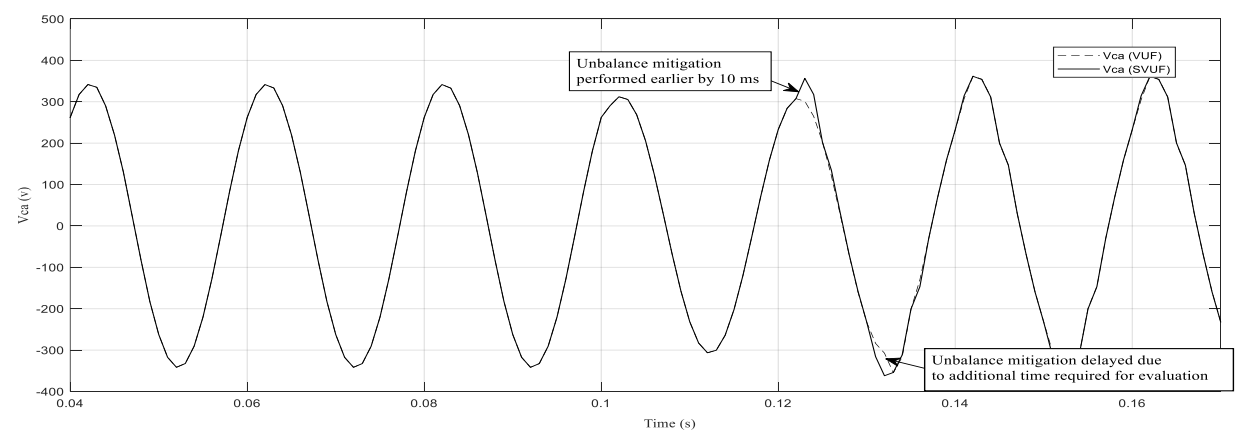

Figure 9. Vca line voltage with NNC considering unbalance evaluation using VUF and SVUF

\section{CONTRIBUTION TO KNOWLEDGE}

Voltage unbalance evaluation was enhanced by using the SVUF, which reduces the time required for evaluation by half while maintaining good accuracy and eliminating the need to use symmetrical component. Table 7 shows a comparison between the proposed RBFNs and NN models and other NNs used in the literature. The total number of parameters includes the parameters of models such as the number of weights and biases used in the NNs. The response time is the time necessary to restore voltage balance excluding the time for voltage unbalance evaluation. In [26] and [27], three NNs with more than one hidden layer were used, resulting in large numbers of parameters causing an excessive increase in computations. A simpler model of one NN with three inputs was proposed in [28]. However, this reduction in the number of networks and inputs was compensated by gradually increasing the number of neurons leading again to large numbers of parameters and excessive computations. Also, the NN suggested has moderate performance during testing, with $\mathrm{R}^{2}$ equals 0.814 . The $\mathrm{NN}$ suggested in [29] has the simplest structure with 163 parameters only and a response time of $40 \mathrm{msec}$.

In this work, a much simpler NN structure is considered, with a total number of parameters equal to 73. Simultaneously, high performance is maintained during the testing phase, with $\mathrm{R}^{2}$ equals 0.976 . In terms of RBFNs, although it has a slightly higher number of parameters, it still provides the best performance in terms of $\mathrm{R}^{2}$ and PRESS $\mathrm{R}^{2}$ leading to higher ability in generating the firing angles with the least VUF. Also, the total response time of TCR is reduced to $30 \mathrm{msec}$. 
Table 7. Comparison between different models

\begin{tabular}{lcccccc}
\hline \multicolumn{1}{c}{ Comparison criterion } & Fuzzy-NN & GSA-NN & NN & PSO-NN & Proposed & Proposed \\
NN & {$[26]$} & {$[27]$} & {$[29]$} & RBN \\
\hline NN Inputs & 3 Reactive & 3 Reactive & 4 Load & 3 Load & 3 Load & 3 Load \\
& power & power & voltages +SV & voltages & voltages & voltages \\
Number of networks & 3 & 3 & 1 & 1 & 1 & 3 \\
Number of neurons in each & $23,13,9$ & 30,30 & 20 & 150 & 10 & - \\
hidden layer & & & & & & \\
Total Number of Parameters & 1620 & 3243 & 163 & 1053 & 73 & 188 \\
$\mathrm{R}^{2}$ for Training & - & - & 0.982 & 0.985 & 0.984 & 1 \\
Response time (msec) & 125 & - & 40 & 57 & 30 & 30 \\
VUF (\%) range & - & - & $3.57-7.54$ & $3.44-6.93$ & $3.28-7.9$ & $3.28-7.9$ \\
\hline
\end{tabular}

\section{CONCLUSION}

The voltage unbalance problem in the electrical power system was tackled through two stages; evaluation and mitigation of voltage unbalance. SVUF was proposed to evaluate system performance, reducing the time required for evaluation from $20 \mathrm{msec}$ to $10 \mathrm{msec}$. Subsequently, voltage-unbalance mitigation was performed through the use of the TCR reactive power compensator. RBFNs and NN were considered to generate the required firing angles of TCR with the three load voltages as the input. The RBFN outperforms existing models with the high ability to fit the data with $\mathrm{R}^{2}$ of 1 while avoiding overfitting with PRESS $\mathrm{R}^{2}$ of 0.999 . In terms of the proposed $\mathrm{NN}$, it has the simplest structure of one network with one hidden layer of ten neurons. Both RBFN and NN show high performance in mitigating voltage unbalance in the range of 3.4 to $7.9 \%$. Furthermore, a reduction in response time of TCR from $40 \mathrm{msec}$ to $30 \mathrm{msec}$ was achieved. The proposed work was verified through a simulation model for the AQSA power system.

\section{ACKNOWLEDGEMENTS} University.

This research work was supported by Palestine Technical University-Kadoorie and Philadelphia

\section{REFERENCES}

[1] D. Mirabbasi, G. Seifossadat, and M. Heidari, "Effect of unbalanced voltage on operation of induction motors and its detection," 2009 International Conference on Electrical and Electronics Engineering-ELECO 2009, 2009, pp. I-189-I-192, doi: 10.1109/ELECO.2009.5355288.

[2] J. Driesen and T. V. Craenenbroeck, "Voltage Disturbances: Introduction to Unbalance," Power Quality Application Guide, Copper Development Association, vol. 5, no. 3, 2002.

[3] J. Ghaeb and J. Chebil, "Prediction of voltage unbalance employing space vector property," International Journal of Engineering Research and Development, vol. 12, no. 12, pp. 65-70, 2016.

[4] B. Bennett, "Unbalanced voltage supply the damaging effects on three phase induction motors and rectifiers," $A B B$ Power Conditioning-Electrification Products Division, 2017, pp. 1-5.

[5] D. Zhang, R. An, and T. Wu, "Effect of Voltage Unbalance and Distortion on the Loss Characteristics of Three-Phase Cage Induction Motor," IET Electric Power Applications, vol. 12, no. 2, pp. 264-270, 2018, doi: 10.1049/iet-epa.2017.0464.

[6] A. I. Adekitan, A. Adewale, and A. Olaitan, "Determining the operational status of a three phase induction motor using a predictive data mining model," International Journal of Power Electronics and Drive Systems (IJPEDS), vol. 10, no. 1, pp. 93103, March 2019, doi: 10.11591/ijpeds.v10.i1.pp93-103.

[7] R. C. F. Gregory, A. C. dos Santos, I. N. Santos, L. Ran, and J. C. de Oliviera, "A practical approach for determining voltage imbalance contributions from suppliers and consumers," International Transactions on Electrical Energy Systems, Wiley, vol. 30, no. 12, p. e12627, September 2020, doi: 10.1002/2050-7038.12627.

[8] A. von Jouanne and B. Banerjee, "Assessment of voltage unbalance," IEEE Transactions on Power Delivery, vol. 16, no. 4, pp. 782-790, October 2001, doi: 10.1109/61.956770.

[9] I. E. C Standard 61000-4-27, “Testing and Measurement Techniques-Unbalance," Immunity Test, August 2000.

[10] "IEEE Standard Dictionary of Electrical and Electronics Terms Sixth Edition," IEEE Std.100-1996, 1996.

[11] "IEEE Standard Test Procedure for Polyphase Induction Motors and Generators," in IEEE Std 112-1991, p. 1, 1991, doi: 10.1109/IEEESTD.1991.114383.

[12] P. Pillay and M. Manyage, "Definitions of Voltage Unbalance," in IEEE Power Engineering Review, vol. 21, no. 5, pp. 49-51, May 2001, doi: 10.1109/MPER.2001.4311362.

[13] "Motors and Generators," ANSI/NEMA Standard MG1-1993, 1993.

[14] S. B. Singh, A. K. Singh, and P. Thakur, "Accurate performance assessment of IM with approximate current unbalance factor for NEMA definition," 2014 16th International Conference on Harmonics and Quality of Power (ICHQP), 2014, pp. 674-678, doi: 10.1109/ICHQP.2014.6842805.

[15] M. Henriques and J. Cormane, "Time Domain Voltage Unbalance Index Based on Second Order Voltage Tensor Theory," 2018 IEEE International Conference on Smart Energy Grid Engineering (SEGE), 2018, pp. 273-278, doi: 10.1109/SEGE.2018.8499508

[16] A. V. Jouanne and B. Banerjee, "Voltage Unbalance: Power qualityissues, related standards and mitigation techniques," Electric Power Research Institute, Palo Alto, CA, EPRI Final Rep., 2000 
[17] H. F. Zhai, M. Yang, B. Chen, and N. Kang, "Dynamic reconfiguration of three-phase unbalanced distribution networks," International Journal of Electrical Power \& Energy Systems, vol. 99, pp. 1-10, July 2018, doi: 10.1016/j.ijepes.2017.12.027.

[18] D. J. R. S. S. Kumar, S. Sasitharan, M. K. Mishra, and B. Kalyan Kumar, "Unbalanced voltage sag correction with dynamic voltage restorer using particle swarm optimization," 2008 Annual IEEE India Conference, 2008, pp. 400-405, doi: 10.1109/INDCON.2008.4768757.

[19] O. P. Mahela and A. G. Shaik, "Topological aspects of power quality improvement techniques: a comprehensive overview," Renewable and Sustainable Energy Reviews, vol. 58, pp. 1129-1142, May 2016, doi: 10.1016/j.rser.2015.12.251.

[20] R. M. Mathur and R. K. Varma, "Thyristor-Based Facts Controllers for Electrical Transmission Systems," John Wiley \& Sons, 2002.

[21] H. Suyono, R. Hasanah, and P. Pranyata, "Optimization of the Thyristor Controlled Phase Shifting Transformer using PSO Algorithm," International Journal of Electrical and Computer Engineering (IJECE), vol. 8, no. 6, pp. 5472-5483, December 2018, doi: 10.11591/ijece.v8i6.pp5472-5483.

[22] S. Suraya, P. Sujatha, and P. B. Kumar, "A Novel Control Strategy for Compensation of Voltage Quality Problem in AC Drives," International Journal of Power Electronics and Drive Systems (IJPEDS), vol. 9, no. 1, pp. 8-16, March 2018, doi: 10.11591/ijpeds.v9n1.pp8-16.

[23] M. T. Esfahani and B. Vahidi, "Development of optimal shunt hybrid compensator based on improving the measurement of various signals," Measurement, vol. 69, pp. 250-263, June 2015, doi: 10.1016/j.measurement.2015.03.021.

[24] M. S. Aziz and A. G. Abdullah, "Hybrid control strategies of SVC for reactive power compensation," Indonesian Journal of Electrical Engineering and Computer Science, vol. 19, no. 2, pp. 563-571, August 2020, doi: 10.11591/ijeecs.v19.i2.pp563-571.

[25] S. Keskes, N. Bouchiba, S. Sallem, L. Chrifi-Alaoui, and M. Kammoun, "Transient stability enhancement and voltage regulation in SMIB power system using SVC with PI controller," 2017 6th International Conference on Systems and Control (ICSC), 2017, pp. 115-120, doi: 10.1109/ICoSC.2017.7958729.

[26] D. B. Kulkarni and G. R. Udupi, "ANN-Based SVC Switching at Distribution Level for Minimal-Injected Harmonics," in IEEE Transactions on Power Delivery, vol. 25, no. 3, pp. 1978-1985, July 2010, doi: 10.1109/TPWRD.2010.2040293.

[27] H. Al-Rubaiey and R. Al-Rubayi, "An artificial neural network based real-time optimal reactive power flow for improving operation efficiency," Int. J. Current Eng. Technol., vol 7, no. 3, pp. 1159-1169, 2017.

[28] M. Alkayyali and J. Ghaeb, "Hybrid PSO-ANN algorithm to control TCR for voltage balancing," IET Generation, Transmission \& Distribution, vol. 14, no. 5, pp. 863-872, March 2020, doi: 10.1049/iet-gtd.2019.1246.

[29] D. M. Ragab, J. Ghaeb, and I. Al-Naimi, "Enhancing the response of thyristor-controlled reactor using neural network," International Transactions on Electrical Energy Systems, vol. 29, no. 12, p. e12137, July 2019, doi: 10.1002/2050-7038.12137.

[30] Z. Chao, Z. Aimin, Z. Hang, B. Yunfei, G. Chujia, and G. Yingsan, "RBF neural networks sliding mode controller design for static var compensator," 2015 34th Chinese Control Conference (CCC), 2015, pp. 3501-3506, doi: 10.1109/ChiCC.2015.7260179.

[31] K. Hongesombut, Y. Mitani, and K. Tsuji, "An adaptive static VAr compensator using genetic algorithm and radial basis function network for enhancing power system stability," 2001 IEEE Porto Power Tech Proceedings (Cat. No.01EX502), 2001, vol. 2, p. 6, doi: 10.1109/PTC.2001.964739.

[32] Y. Guo, K. Li, Z. Yang, J. Deng, and D. M. Lverty, "A novel radial basis function neural network principal component analysis scheme for PMU-based wide-area power system monitoring," Electric Power Systems Research, vol. 127, pp. 197-205, October 2015, doi: 10.1016/j.epsr.2015.06.002.

[33] S. Marx and D. Bender, "An Introduction to Symmetrical Components, System Modeling and Fault Calculation," 34th Annual HANDS-ON Relay School, Washington State Univ. Washington, USA, 2016.

[34] V. Peter, "Vector Control of AC Machines," New York: Oxford University Press, 1990

[35] "Model-Based Calibration Toolbox for use with MATLAB and Simulink," 2nd ed. Natick: Math Works, 2004.

\section{BIOGRAPHIES OF AUTHORS}

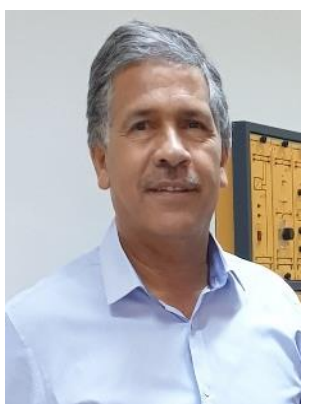

Jasim Ghaeb (iD ISI SC P is a professor of power systems and he is currently with the Department of Alternative Energy Technology, Faculty of Engineering and Technology, Philadelphia University, Jordan. He received a Ph.D. degree from the University of Bradford, U.K, 1989 in electrical power systems- control and power electronics. Ghaeb is with 30 years of experience in teaching, research, and administration. Areas of research include reactive power control, artificial intelligence, and heuristic algorithms-based power system control, and embedded systems and data integrity. He can be contacted at email: dmrafat@gmail.com.

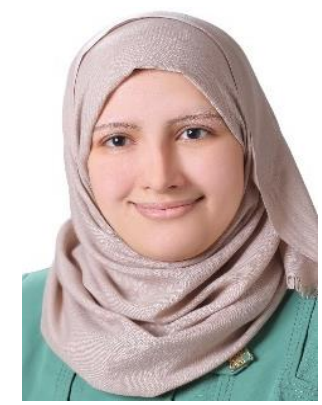

Dana Ragab (iD SA SC P is a lecturer at mechatronics engineering department, Palestine Technical University, Palestine. She received MSc. degree from Philadelphia University in Jordan, 2018 in Mechatronics Engineering. Areas of research include mechatronics system design, unbalanced power system control, and artificial intelligence applications. She can be contacted at email: gaebja@yahoo.com. 